\title{
COMPARISON OF HEAT SHOCK PROTEIN GENE (HSP70-1) SEQUENCE IN ARADI AND DAMASCUS GOAT BREEDS (Capra hircus) RAISED UNDER HEAT STRESS CONDITIONS
}

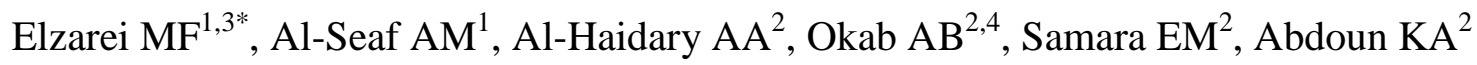 \\ ${ }^{1}$ Department of Animal P roduction and Breeding, College of Agriculture and Veterinary Medicine, Qassim University, Qassim, Saudi Arabia \\ ${ }^{2}$ Department of Animal Production, College of Food and Agriculture Sciences, King Saud University, Riyadh, Saudi Arabia \\ ${ }^{3}$ Department of Animal Production, Faculty of Agriculture, Suez Canal University, Ismailia, 41522, Egypt \\ ${ }^{4}$ Department of Environmental Studies, Institute of Graduate Studies and Research, Alexandria University, Egypt
}

Received - March 09, 2017; Revision - March 29, 2017; Accepted - March 30, 2017

Available Online - August 31, 2017

DOI: http://dx.doi.org/10.18006/2017.5(Spl-1-SAFSAW).S63.S67

\section{KEYWORDS}

Goat

HSP70-1

DNA - Sequence

Aradi

Damascus

\begin{abstract}
Ten animals from two goat breeds (Aradi and Damascus) raised under heat stress conditions in Saudi Arabia were used in this study to compare the sequence of Heat Shock Proteins-70 gene (HSP70-1) between Aradi and Damascus goats with the reported one of Yunnan black goat (Capra hircus). From the sequence of the above mentioned three breeds Aradi, Damascus and Yunnan, it could be identify that, Damascus goats are more mutant to HSP70-1 gene sequence than other breeds. The results of study also showed an addition of A, $\mathrm{T}$ and G nucleotides in positions 935 (AGAAAGGCTC), 984 (GGTTCCTGGT) and 997 (GGGGGGGCTC) in Damascus Goats. However, Aradi goats did not differ in these positions from Yunnan black goats (AGAAAGGCTC, GGTTCCTGGT and GGGGGGGCTC). It could be concluded that HSP70-1 gene sequence varied between Aradi and Damascus breeds and Damascus breed showed more mutation in DNA sequence in 3 positions, Aradi breed did not show any variation in relation to that published for Capra hircus.
\end{abstract}

* Corresponding author

E-mail: zray@qu.edu.sa (Elzarei MF)

Peer review under responsibility of Journal of Experimental Biology and Agricultural Sciences.

Production and Hosting by Horizon Publisher India [HPI] (http://www.horizonpublisherindia.in/).

All rights reserved.
All the article published by Journal of Experimental Biology and Agricultural Sciences is licensed under a Creative Commons Attribution-NonCommercial 4.0 International License Based on a work at www.jebas.org.

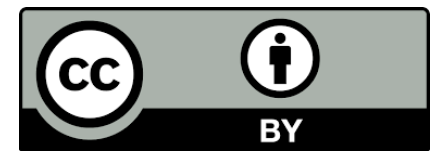




\section{Introduction}

In response to environmental stressors, farm animals generate certain reactions at cellular level, like heat stress response, in which the cell produce a series of proteins called heat shock proteins (HSP).Heat shock proteins are family of proteins that are classified based on their molecular weight including the 70kilodalton heat shock proteins (HSP70s). The HSP70s play a crucial role in the cell's machinery for protein folding and cells stress protection. Specifically, HSP70 assists the folding of newly formed polypeptide chains, acts as a molecular chaperone and mediate the repair and degradation of altered or denatured proteins. Heat stress in mammals induces cellular changes in gene expression and in the activity of expressed proteins, resulting in cell stress response (Lindquist, 1986; Jaattela, 1999). According to Luengrattana et al. (2000), one of the regions containing two tandem arrays HSP70 sequences was specified as HSP70-1 and HSP70-2. The other two regions containing a single HSP70 sequences were specified as HSP70-3 and HSP70-4. The unrestrained HSP70 expression is principally a result of the transcription of the HSP70-1 locus (Christians et al., 1997). Further, Ramunno et al. (2005) stated that characterization of HSP70-1 locus is playing an important role to pediment phylogenic relationship among specific species. Accordingly, to increase our knowledge about HSP70 gene and to provide some helpful database for goat breeding, sequence determination and characterization of goat HSP70-1 gene is important and targeted in the current study. Aradi goat breed is one of the most important goat breeds in Saudi Arabia, it is well-adapted to local environment and has been crossed with imported breeds like Damascus (from Syria) to improve its production and reproduction. It is well known that, genetic improvement of any livestock breed depends on the identification of animals that are capable of transmitting their desirable characteristics to their offspring. This study describes the results of comparison of the single HSP70 sequence (HSP 70-1) of Aradi and Damascus goat with reference to Yunnan black goat (Gade et al., 2010 and Dong et al., 2013) as a method of evaluation.

\section{Material and Methods}

This study carried out at biotechnology Laboratory, Department of Animal Production and Breeding, College of Agriculture and
Veterinary Medicine, Qassim University, Kingdom of Saudi Arabia. Five animals from each breed (Aradi and Damascus), raised in animal experimental farm of Qassim University used in this study. DNA of these two breeds was taken from the whole fresh blood samples using EDTA as anticoagulant. Blood samples were kept in ice box until reaching the laboratory within 2 hours and then the DNA extraction began. DNA from whole blood sample isolated using ILLUSTRA blood mini spin kit (GE Life Sciences, USA). Then DNA samples were checked for quantity and quality using Thermo Scientific ${ }^{\mathrm{TM}}$ NanoDropUSA). Primers used to amplify the selected gene (Table 1) taken according to Gade et al. (2010)

Table 1 Primers used in the present study

\begin{tabular}{|ccc|} 
References & Name & Sequence \\
\hline $\begin{array}{r}\text { Gade et } \\
\text { al., 2010 }\end{array}$ & HSP70-1-F & 5_ATGGCGAAAAACATGGCTATC-3 \\
\cline { 2 - 3 } & HSP70-1-R & 5_CTAATCCACCTCCTCAAT-3 \\
\hline
\end{tabular}

\subsection{Herd nutrition and management}

Animal used in this study were housed in semi-shaded/open front barn. Goats were fed on a commercial pre-formulated total mixed ration (TMR, Alwafi-ARASCO-KSA). According to the manufacturer's specifications, the TMR consisted of alfalfa hay, barley, corn, wheat bran, soybean meal and crust, molasses, vitamins and minerals; and contained on DM basis $13 \%$ crude protein, $2 \%$ ether extract, $9 \%$ crude fiber, $8 \%$ ash, $1 \%$ calcium, $0.5 \%$ phosphorus, $0.7 \%$ sodium chloride, and $2.95 \mathrm{Mcal} / \mathrm{kg}$ digestible energy. The average high temperature through summer months when samples were collected was $46^{\circ} \mathrm{C}$ and $43^{\circ} \mathrm{C}$, for August and September, respectively. The average low temperatures through these two months were $29^{\circ} \mathrm{C}$ and $26^{\circ} \mathrm{C}$, for August and September, respectively.

\subsection{Sequencing Protocol for required genes}

The reaction mixtures were prepared according to Thermo Scientific protocol. Briefly, the terminator ready reaction consisted of $(8.0 \mu \mathrm{L})$, template $(20 \mu \mathrm{g})$, primer $(3.2 \mathrm{pmol})$, deionized water to volume $(20 \mu \mathrm{L})$ the mixture was then mixed well and spanned briefly, according to amplification conditions presented in table (2). Amplification through Veriti ${ }^{\circledR}$ Thermal Cycler Protocol used for Purification using sequential addition of

Table 2 PCR amplification conditions for used primers

\begin{tabular}{|lcccccc|}
\hline PCR Product & & \multicolumn{2}{c|}{ Amplification conditions } \\
\hline & Pre-denature & Cycles of reaction & Denature & Annealing & Extension \\
\hline HSP70 (HSP) & $94^{\circ} \mathrm{C}, 3 \mathrm{~min}$ & 35 & $94^{\circ} \mathrm{C}, 1 \mathrm{~min}$ & $49^{\circ} \mathrm{C}, 45 \mathrm{sec}$ & $72^{\circ} \mathrm{C}, 2.20 \mathrm{~min}$ & $72^{\circ} \mathrm{C}, 10 \mathrm{~min}$ \\
\hline
\end{tabular}

Journal of Experimental Biology and Agriculture Science

http://www.jebas.org 
the BigDyeXTerminator Purification Kit reagents and ran in 3500 DNA Analyzer. Data were analyzed using Applied Biosystems DNA Sequencing Analysis Software Version 5.1.

\section{Results}

From the below sequence of the two breeds Aradi and Damascus the following differences could be specified, three nucleotides A, $\mathrm{T}$ and $\mathrm{G}$ were identified to the sequence of Damascus goat in positions 935, 984 and 996, respectively (Figure 1) but not appeared in Aradi sequence (Figure 2).

As presented in Figures 1 and 2 for Damascus and Aradi breeds, it can be deduced that the two breeds did not show any differences in either forward or reverse strand except in positions from 935 to 996. Moreover, no differences could be found between Aradi breed HSP70.1 sequence compared with that reported in Yunnan breed (Dong et al., 2013) and Yunnan black breed (Gade et al., 2010).

\section{Discussion and Conclusions}

The current study was aimed to compare the single HSP70 sequence of Aradi and Damascus goats with that published for Yunnan black and Yunnan goat. HSPs expression has been widely used to characterize the cellular response to heat stress. The sequences of HSP70-1 gene in small ruminants (Goat and sheep) are well maintained and are also matches with many other species. Goats, as other mammals, respond to heat stress at the cellular level by synthesizing HSPs, which help protect cells from the deleterious effects induced by heat stress (Welch, 1992; Morimoto

ID EMBOSS_001; SV 1; Tinear; unassigned DNA; STD; UNC; 1891 BP.

SQ Sequence $1891 \mathrm{BP} ; 433 \mathrm{~A} ; 551 \mathrm{C} ; 638 \mathrm{G} ; 269 \mathrm{~T} ; 0$ other; TTGCTTGAGG TACGTCTTCA CGTTGTGTGA TTGTTCCTGC GCGAGCATAG GATGTAAGAA 60 TCATCGCCAA CGACCAGGGC AACCGCACCA CCCCCAGCTA CGTGGCTTTC ACCGATACCG 120 AGCGGCTCAT CGGCGATGCA GCCAAGAACC AGGTGGCGCT GAACCCGCAG AACACCGTGT 180 TCGACGCGAA GCGGCTGATC GGCCGCAAGT TCGGCGACCC GGTGGTGCAG TCGGACATGA 240 AGCACTGGCC TTTCCGCGTG ATCAACGACG GAGACAAGCC TAAAGTGCAG GTGAGCTACA 300 AGGGGGAGAC CAAGGCGTTC TACCCAGAGG AGATCTCGTC GATGGTGCTG ACCAAGATGA 360 AAGAGATCGC CGAGGCGTAC CTGGGCCACC CGGTGACCAA CGCGGTGATC ACCGTGCCGG 420 CCTACTTCAA CGACTCGCAG CGGCAGGCCA CCAAGGACGC GGGGGTGATC GCGGGGCTGA 480 ACGTGCTGAG GATCATCAAC GAGCCCACGG CCGCCGCCAT CGCCTACGGC CTGGACCGGA 540 CGGGCAAGGG GGAGCGCAAC GTGCTCATCT TTGACCTGGG CGGGGGCACG TTCGACGTGT 600 CCATCCTGAC GATCGACGAC GGCATCTTCG AGGTGAAGGC CACGGCCGGG GACACGCACC 660 TGGGCGGGGA GGACTTCGAC AACAGGCTGG TGAACCACTT CGTGGAGGAG TTCAAGAGGA 720 AGCACAAGAA GGACATCAGC CAGAACAAGC GGGCCGTGAG GCGGCTGCGC ACGGCGTGCG 780 AGCGGGCCAA GAGGACCTTG TCGTCCAGCA CCCAGGCCAG CCTGGAGATC GACTCCCTGT 840 TCGAGGGCAT CGACTTCTAC ACGTCCATCA CCAGGGCACG GTTCGAGGAG CTGTGCTCCG 900 ACCTGTTCCG GAGCACCCTG GAGCCCGTGG AGAAAGGCTC TACGCGACGC CAAGCTGGAC 960 AAGGCCCAGA TCCACGACCT GGTTCCTGGT GGGGGGGCTC CACCCGCATC CCCAAAGTGC 1020 AGAAGCTGCT GCAGGACTTC TTCAACGGGC GCGACCTCAA CAAGAGCATC AACCCGGACG 1080 AGGCGGTGGC ATACGGGGCG GCGGTGCAGG CGGCCATCCT GATGGGGGAC AAGTCGGAGA 1140 ACGTGCAGGA CCTGCTGCTG CTGGACGTGG CTCCCCTGTC GCTGGGACTG GAGACGGCCG 1200 GAGGCGTGAT GACTGCCCTG ATCAAGCGCA ACTCCACCAT CCCCACGAAG CAGACGCAGA 1260 TCTTCACCAC CTACTCGGAC AACCAGCCGG GCGTGCTGAT CCAGGTGTAC GAGGGCGAGA 1320 GGGCCATGAC TCGGGACAAC AACCTGCTGG GGCGCTTCGA GCTGAGCGGC ATCCCGCCGG 1380 CCCCGCGGGG GGTGCCCCAG ATCGAGGTGA CCTTCGACAT CGACGCCAAT GGCATCCTGA 1440 ACGTCACGGC CACGGACAAG AGCACGGGCA AGGCCAACAA GATCACCATC ACCAACGACA 1500 AGGGCCGGCT GAGCAAGGAG GAGATCGAGC GCATGGTGCA GGAGGCGGAG AAGTACAAGG 1560 CAGAGGACGA GGTCCAGCGC GAGAGGGTGT CTGCCAAGAA CGCGCTGGAG TCGTACGCCT 1620 TCAACATGAA GAGCGCCGTG GAGGATGAGG GGCTGAAGGG CAAGATCAGC GAGGCGGACA 1680 AGAAGGTGGT GCTGGACAAG TGCCAGGAGG TGATTTCCTG GCTGGACGCC AACACCTTGG 1740 CGGAGAAGGA CGAGTTTGAG CACAAGAGGA AGGAGCTGGA GCAGGTGTGT AACCCCATCA 1800 TCAGCAGACT GTACCAGGGG GCGGGCGTAC CCCGGGGCTG CGACGGCTTA TGGGGCTACA 1860 GCCCCAAACG AATCAACATA CGAACTAAAC C 
ID EMBOSS_001; SV 1; 1inear; unassigned DNA; STD; UNC; 1887 BP.

SQ Sequence $1887 \mathrm{BP} ; 440 \mathrm{~A} ; 548 \mathrm{C} ; 637 \mathrm{G} ; 262 \mathrm{~T} ; 0$ other; GAGTGCCTCC TACGTCTTCA AGTTGGTCGG TGTTCCAGCG CGAGCAAGGA TGTAAGAATC 60 ATCGCCAACG ACCAGGGCAA CCGCACCACC CCCAGCTACG TGGCTTTCAC CGATACCGAG 120 CGGCTCATCG GCGATGCAGC CAAGAACCAG GTGGCGCTGA ACCCGCAGAA CACCGTGTTC 180 GACGCGAAGC GGCTGATCGG CCGCAAGTTC GGCGACCCGG TGGTGCAGTC GGACATGAAG 240 CACTGGCCTT TCCGCGTGAT CAACGACGGA GACAAGCCTA AAGTGCAGGT GAGCTACAAG 300 GGGGAGACCA AGGCGTTCTA CCCAGAGGAG ATCTCGTCGA TGGTGCTGAC CAAGATGAAA 360 GAGATCGCCG AGGCGTACCT GGGCCACCCG GTGACCAACG CGGTGATCAC CGTGCCGGCC 420 TACTTCAACG ACTCGCAGCG GCAGGCCACC AAGGACGCGG GGGTGATCGC GGGGCTGAAC 480 GTGCTGAGGA TCATCAACGA GCCCACGGCC GCCGCCATCG CCTACGGCCT GGACCGGACG 540 GGCAAGGGGG AGCGCAACGT GCTCATCTTT GACCTGGGCG GGGGCACGTT CGACGTGTCC 600 ATCCTGACGA TCGACGACGG CATCTTCGAG GTGAAGGCCA CGGCCGGGGA CACGCACCTG 660 GGCGGGGAGG ACTTCGACAA CAGGCTGGTG AACCACTTCG TGGAGGAGTT CAAGAGGAAG 720 CACAAGAAGG ACATCAGCCA GAACAAGCGG GCCGTGAGGC GGCTGCGCAC GGCGTGCGAG 780 CGGGCCAAGA GGACCTTGTC GTCCAGCACC CAGGCCAGCC TGGAGATCGA CTCCCTGTTC 840 GAGGGCATCG ACTTCTACAC GTCCATCACC AGGGCACGGT TCGAGGAGCT GTGCTCCGAC 900 CTGTTCCGGA GCACCCTGGA GCCCGTGGAG AAGGCTCTAC GCGACGCCAA GCTGGACAAG 960 GCCCAGATCC ACGACCTGGT CCTGGTGGG GGCTCCACCC GCATCCCCAA AGTGCAGAAG 1020 CTGCTGCAGG ACTTCTTCAA CGGGCGCGAC CTCAACAAGA GCATCAACCC GGACGAGGCG 1080 GTGGCATACG GGGCGGCGGT GCAGGCGGCC ATCCTGATGG GGGACAAGTC GGAGAACGTG 1140 CAGGACCTGC TGCTGCTGGA CGTGGCTCCC CTGTCGCTGG GACTGGAGAC GGCCGGAGGC 1200 GTGATGACTG CCCTGATCAA GCGCAACTCC ACCATCCCCA CGAAGCAGAC GCAGATCTTC 1260 ACCACCTACT CGGACAACCA GCCGGGCGTG CTGATCCAGG TGTACGAGG CGAGAGGGCC 1320 ATGACTCGGG ACAACAACCT GCTGGGGCGC TTCGAGCTGA GCGGCATCCC GCCGGCCCCG 1380 CGGGGGGTGC CCCAGATCGA GGTGACCTTC GACATCGACG CCAATGGCAT CCTGAACGTC 1440 ACGGCCACGG ACAAGAGCAC GGGCAAGGCC AACAAGATCA CCATCACCAA CGACAAGGGC 1500 CGGCTGAGCA AGGAGGAGAT CGAGCGCATG GTGCAGGAGG CGGAGAAGTA CAAGGCAGAG 1560 GACGAGGTCC AGCGCGAGAG GGTGTCTGCC AAGAACGCGC TGGAGTCGTA CGCCTTCAAC 1620 ATGAAGAGCG CCGTGGAGGA TGAGGGGCTG AAGGGCAAGA TCAGCGAGGC GGACAAGAAG 1680 GTGGTGCTGG ACAAGTGCCA GGAGGTGATT TCCTGGCTGG ACGCCAACAC CTTGGCGGAG 1740 AAGGACGAGT TTGAGCACAA GAGGAAGGAG CTGGAGCAGG TGTGTAACCC CATCATCAGC 1800 AGACTGTACC AGGGGGCGGG CGTGACTCGC CGAGAAGACA GCGACTGGCT ATGGAGCACA 1860 GAACCTCTAA ACGATGACAA AAAAATA

Figure 2: Complete sequence of HSP1 gene, in Aradi Breed

et al., 1994).It has been proven that summer heat stress increases the mRNA expression of HSPs in goats either in tropical or temperate regions which might play a crucial role in resistance to hot environmental conditions (Dangi et al., 2012). However, the role of individual members of HSP70 family genes under heat stress conditions need more studies in different areas and breeds of goats. The current study revealed the expression pattern of individual member genes of Aradi goat (a local breed in Saudi Arabia) HSP70 family in comparison with Damascus goat (a Syrian breed that commonly crossed with Aradi breed to improve production and reproduction). A preliminary focus in this gene was a part of a big project to study the effect of exposure to heat stress on mRNA expression levels that encoded both HSP70 and
HSP90 proteins. HSP70 Expression of genes can be utilized as a marker for heat tolerance in various species, and collected produced data will have valuable effect in improvement of technique to be used in Animal breeding systems for adapting to challenges of environmental changes.

As mentioned in results section, Aradi breed did not show any variation in HSP70-1sequence compare with that reported by Gade et al. (2009) in Yunnan black goat breed and Dong et al., (2013) in Yunnan breed. On the other hand, Damascus breed showed variation in HSP70-1 sequence compared with Yunnan or Yunnan black breeds (Gade et al., 2010; Dong et al., 2013).Three nucleotides A, T and G were identified in HSP70-1 sequence in 
Damascus goat in positions 935, 984 and 996, respectively but did not identified in Aradi, Yunnan or Yunnan black goat breeds. It could be concluded that the Sequence data and typing results of HSP70-1 gene varied between Aradi and Damascus breeds which can assist as a method for goat herd's evaluation. In addition to that, Damascus breed showed mutation in DNA sequence in 3 positions, Aradi breed did not show any variation in relation to that published for $C$. hircus.

\section{Acknowledgements}

This Project was funded by the National Plan for Science, Technology and Innovation (MAARIFAH), King Abdulaziz City for Science and Technology, Kingdom of Saudi Arabia, Award Number (12-AGR2540-02)

\section{Conflict of interest}

Authors would hereby like to declare that there is no conflict of interests that could possibly arise.

\section{References}

Christians E, Michel E, Adenot P, Mezger V, Rallu M, Morange M, Renard JP (1997) Evidence for the involvement of mouse heat shock factor 1 in the atypical expression of the HSP70.1 Heat shock gene during mouse zygotic genome activation. Molecular and Cellular Biology 17 : 778-788.

Dangi SS, Gupta M, Maurya D, Yadav VP, Panda RP, Singh G, Mohan NH, Bhure SK, Das BC, Bag S, Mahapatra R, Sharma GT, Sarkar M (2012) Expression profile of HSP genes during different seasons in goats Capra hircus. Tropical Animal Health and Production 44 : 1905-1912.

Dong Y, Xie M, Jiang Y, Xiao N, Du X, Zhang W, Tosser-Klopp G, Wang J, Yang S, Liang J, Chen W, Chen J, Zeng P, Hou Y, Bian C, Pan S, Li Y, Liu X, Wang W, Servin B, Sayre B, Zhu B, Sweeney D, Moore R, Nie W, Shen Y, Zhao R, Zhang G, Li J, Faraut T, Womack J, Zhang Y, Kijas J, Cockett N, Xu X, Zhao S, Wang J, Wang W (2013) Sequencing and automated wholegenome optical mapping of the genome of a domestic goat (Capra hircus). Nature Biotechnology 31+ : 135-141. DOI: http://dx.doi.org/doi:10.1038/nbt.2478
Gade N, Mahapatra RK, Sonawane A, Singh VK, Doreswamy R, Saini M (2010) Molecular Characterization of Heat Shock Protein 70-1 Gene of Goat (Capra hircus). Molecular Biology International Article ID 108429, 7 pages. DOI: http://dx.doi.org/doi:10.4061/2010/108429

Gade NE, Mahapatra RK, Singh VK, Ramesh D, Saini M (2009) Cloning and characterization of cDNA encoding heat shock protein (HSP) 70.1 from Capra hircus, NCBI last access: $1^{\text {st }}$ March 2017, available on https://www.ncbi.nlm.nih.gov/nuccore/238801230 access on 25th May 2017.

Jaattela M (1999) Heat shock proteins as cellular lifeguards. Annals of Medicine 31 : 261-271. DOI: http://dx.doi.org/10.3109/07853899908995889

Lindquist S (1986) The heat-shock response. Annual Review of Biochemistry 55:1151-1191. DOI: http://dx.doi.org/10.1146/annurev.bi.55.070186.005443.

Luengrattana Y, Kohara S, Hara H, Mukoyama H, Hanzawa K, Watanabe S (2000) Complete Nucleotide Sequence of a Shiba Goat (Capra hircus) Heat-shock Protein 70-3 (HSP70-3) Gene. Animal Science Journal 71 : 371-376. DOI: http://doi.org/10.2508/chikusan.71.371.

Morimoto RI, Tissieres A, Georgopoulos C (1994) Progress and perspectives on the biology of heat shock proteins and molecular chaperones In: Morimoto RI, Tissieres A, Georgopoulos C (Eds), The Biology of Heat Shock Proteins and Molecular Chaperones. Cold Spring Harbour Laboratory Press, Cold Spring Harbour, pp. $1-30$.

Ramunno L, Cosenza G, Rando A, Pauciullo A, Illario R, Gallo D, Di Bernardino D, Masina P (2005) Comparative analysis of gene of goat CSN1S1 $\mathrm{F}$ and $\mathrm{N}$ alleles and characterization of CSN1S1 transcript variants in mammary gland. Gene 345 : 289299. DOI: http://dx.doi.org/10.1016/j.gene.2004.12.003

Welch WJ (1992) Mammalian stress response: cell physiology, structure/function of stress proteins, and implications for medicine and disease. Physiological Reviews 72:1063-1081. 